\title{
Research on Route Guidance Algorithm for Transportation System
}

\author{
Mao Yongming ${ }^{*}$ \\ Information and Control Engineering Academy, \\ Shenyang Jianzhu University \\ Shen Yang, China \\ e-mail: arnold0110@ sina.com \\ * Corresponding Author \\ Zhang Dongwei \\ School of Information \& Control Engineering \\ Shenyang Jianzhu University \\ Shenyang China \\ QiNing \\ The School Library \\ Shenyang Jianzhu University \\ Shenyang China \\ Kan Fenglong \\ School of Information \& Control Engineering \\ Shenyang Jianzhu University \\ Shenyang, China
}

\author{
Wang Xin \\ School of Information \& Control Engineering \\ Shenyang Jianzhu University \\ Shenyang China \\ Wang Changtao \\ School of Information \& Control Engineering \\ Shenyang Jianzhu University \\ Shenyang, China \\ Kan Hongliang \\ School of Information \& Control Engineering \\ Shenyang Jianzhu University \\ Shenyang, China \\ Wang Bin \\ Northeastern University at Qinhuangdao \\ Qinhuangdao, China
}

\begin{abstract}
Objective: The Route Guidance System (RGS) is an effective method which can raise the quality of transport service, enhance the driving efficiency of the vehicle, decrease the pressure of the crowed traffic and reduce the traffic accidences. RGS can offer the traveler the most satisfying route dynamically based on the traveler's original demand (OD), or help the traveler find the optimal route from the beginning to the density through the traffic information. Methods: The method of the RGS is discussed deeply in this paper. Moreover, a new route guidance method, based on the genetic algorithm, which is a means of improvement to the standard genetic algorithm (SGA), is put forward. Improved route guidance method is proposed with rectangular restricted searching area. Results: To probe into the technology of the RGS, a large number of experiments combining with the analysis of the results have been carried on, which consider the traffic network as the object consisting of partial route net of Shengyang. It is indicated by simulation experiments that the effectiveness of the method can be $99.8 \%$ and the accuracy can be $97.6 \%$. Conclusion: It can fulfill the real time requiring of route guidance for the large route net, and have definite applications value.
\end{abstract}

Keyword-Intelligent Transportation System, Route Guidance System, Genetic Algorithm, Rectangular Restricted Searching Area Algorithm, the Selection of Optimal Route.

\section{INTRODUCTION}

At present, due to lack of efficient dynamic route guidance system, urban traffic congestion and traffic accidents are more and more seriously affects the world's big cities [1]. With the rapid development of our country transportation enterprise, how to study a set of efficient route guidance system, so as to avoid blind travel caused by the traffic jam, for our country's national economic construction has the important practical significance and economic value [2-4]. In view of this, this paper studies the optimal dynamic path selection method based on rectangle search algorithm combined with genetic algorithm to improve.

\section{THE SIMULATION OF OPTIMAL PATH SELECTION ALGORITHM BASED ON SGA}

Chromosome coding with binary coding and symbol encoding, real number encoding is a special case of the symbol encoding. Due to the optimal path selection algorithm is the genetic network node, and the order of these nodes is required in the path, so this article took the ordered real number encoding [5-7].

This paper applies the optimal path selection of chromosome selection method. First of all to the generation of chromosomes after roulette wheel select, crossover and mutation for the next generation chromosomes [8-9]. In previous generations of genetic calculation, a large number of chromosomes is not reasonable, therefore, reasonable chromosome can be done in this generation path, instead of the next generation of the number of genes in a little unreasonable chromosome; If this generation does not reasonable chromosome, not to replace. When genetic computing is going to algebra, chromosome most of them are reasonable. 


\section{A. Network parameter selection}

As shown in figure 1, this paper takes some road network simulation as the research object, using genetic algorithm to calculate the optimal path. The network contains 30 nodes, 94 roads.

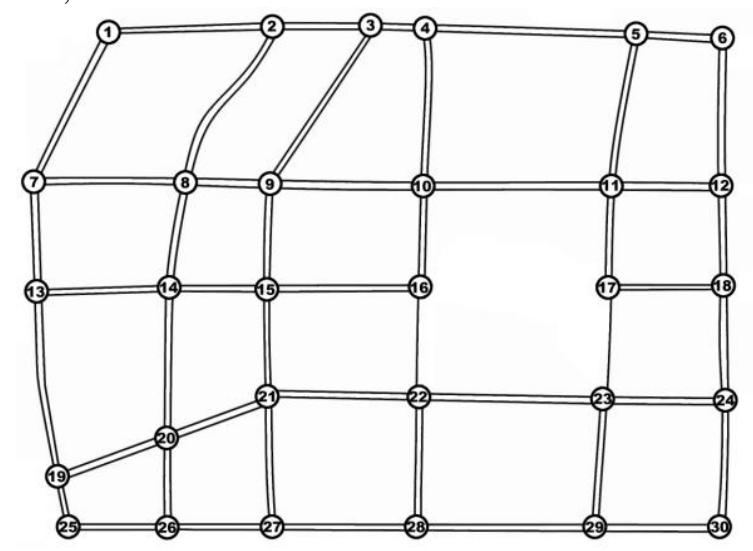

Figure 1. The simulation models for road network

B. Algorithm process of optimal path selection algorithm based on $S G A$

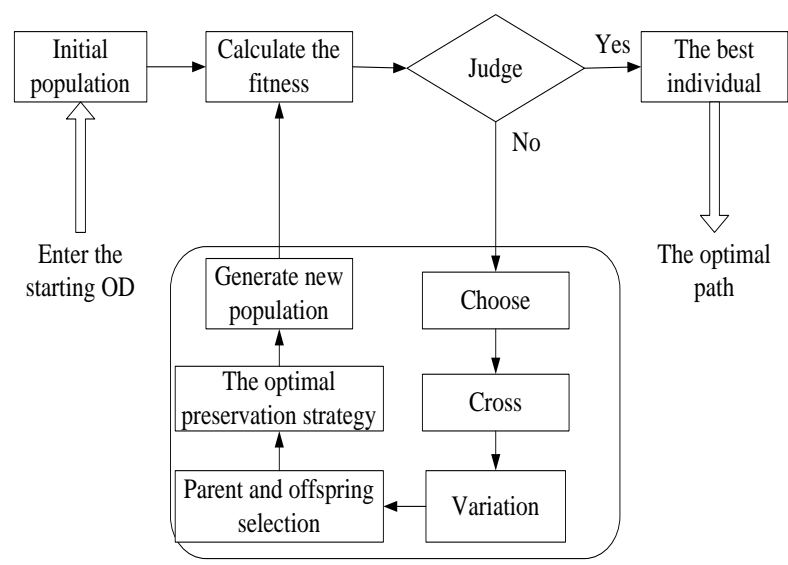

Figure 2. The diagram of optimal vehicle routing al gorithm based on SGA

\section{The determination of the genetic algorithm parameters}

1) Chromosome coding length stringlength

In computing, because the network nodes is 30 , so the length of the chromosome coding stringlength is 30 ;

2) Population size popsize

Through times of simulation test and verify, when the population size is 400 , the performance of the optimal path selection algorithm is best.

3) Select rate Pc and mutation rate Pm

Because in this paper, the design of crossover operator and mutation operator can promote the increase of the population chromosome fitness, therefore, $\mathrm{Pc}, \mathrm{Pm}$ values can be slightly bigger. $\mathrm{Pc}=0.4$ and $\mathrm{Pm}=0.6$.

\section{4) Termination conditions}

In this paper, the genetic algorithm is the termination conditions of termination of the algebraic $\mathrm{T}$ reaches a given value. Through many simulation experiments show that when more than 30 genetic algebra, chromosome fitness basic don't change, therefore, $\mathrm{T}=$ 30 .

\section{The results of simulation and analysis}

For the optimal vehicle routing algorithm based on SGA, the MATLAB language is used for the simulation experiment, the simulation calculation 586 times. For most of the genetic algorithm can accurately find the shortest path. For any OD pairs, a path through the calculation of the genetic algorithm to find the time spent is less than 3 seconds, as a result, the optimal path selection method based on genetic algorithm can satisfy the real-time demand in route guidance of intelligent traffic.

\section{THE IMPROVED DYNAMIC OPTIMAL PATH SELECTION ALGORITHM}

\section{A. Restricted areas search algorithm}

Analyze some characteristics of road network, road route design process in order to convenient traffic and reduce the cost, usually in without obstacle (mountains, rivers, etc.) routes along a straight line to build as much as possible. Online to select any two points on the road as a starting point and end point, according to the observation, can have the following two conclusions:

(1) If the two points in the same section of the moment, so the route that the shortest distance between two points on the road.

(2) If in different sections, the shortest distance between two points of the line should be better than other feasible route in the straight line between two points.

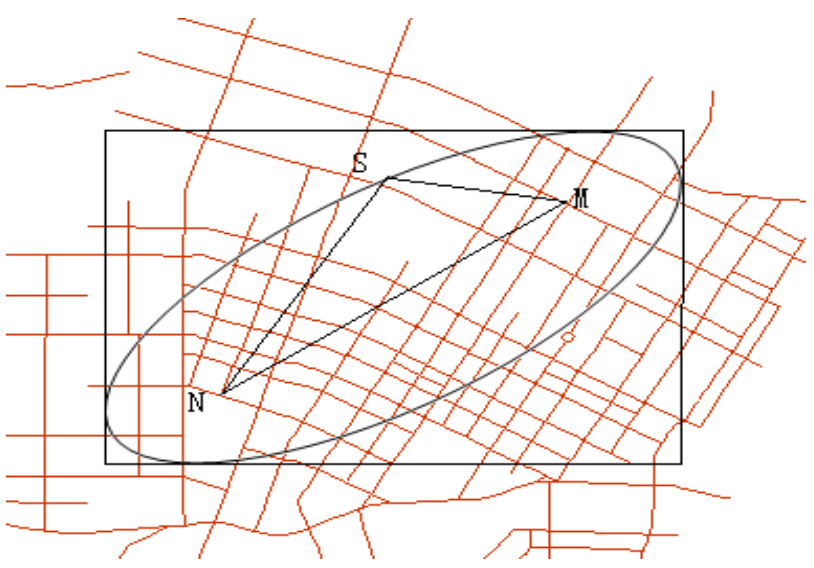

Figure 3. Set search area in the road network

According to the characteristics of road network, Stig Nordbeck [10] put forward such as elliptic search algorithm is proposed, as shown in figure 3, after the end and the starting point in determining the $\mathrm{M} \mathrm{N}$ due to its own characteristics of road network, the shortest path will there is a corresponding roughly the distance limit, to the end and the starting point as the focus, in the shortest path to the corresponding critical distance for long axis.

Because whether sections within the ellipse is trivial, Lu Feng [11] put forward elliptic search box algorithm, and rectangle restricted areas search algorithm is proposed. The rectangle restricted areas search algorithm is out of 
ellipse is used to determine whether a circumscribed rectangle as the search area, simplify the process to determine whether a line segment in the region, so as to improve the efficiency. Either elliptical or rectangular algorithm is the key to get a proper roughly the limit of the shortest path distance. Assuming that the linear distance between two points is $\mathrm{P}$, the corresponding critical distance roughly for $\mathrm{Q}$, defined the scaling factor $\mathrm{t}=\mathrm{P} / \mathrm{Q}$ [5].

In this paper, to determine whether a road in the area of the calculation principle is to calculate whether the beginning and end of the road in the area.

\section{B. The realization of the improved dynamic optimal path selection algorithm}

Because the route guidance system in the network topology environment is complicated, the dynamic optimal path algorithm based on genetic algorithm (GA) is completed the final process of the search process of complex than the original algorithm. First, genetic algorithm and the value of the rights of basic parameters are preset fixed, also need to determine the scope of the search, and dynamic real-time optimal path algorithm, as well as complex calculation steps of weight determination process, also ready to finally determine a range search process, the last to use genetic algorithm to complete the final optimization calculation. In addition, because the dynamic route guidance system and some real-time traffic information input, possibly while computing the optimal path at one point is correct, but, if there is some sudden accidents, the results may need to recalculate.

The algorithm process of the improved optimal dynamic path selection:

(1) According to the user input starting OD, determine the position of the two points on the map.

(2) Calculate the linear distance between two points P, is more than using rectangle area limit distance Pmax search algorithm, is go to step (4);Otherwise the step (7).

(3) According to the linear distance between two points to determine the scaling factor $\mathrm{Pt}$.

(4) According to the value of P and t, calculation of rectangular limits the search area.

(5) On the electronic map search in rectangular network nodes within the restricted areas, and based on the point within the restricted areas of road, the road in the principles of regional development, limiting search nodes and calculate the number of nodes.

(6) According to the step (5) nodes to determine the population size of hybrid algorithm, the length of the chromosome, loop algebra dynamic parameters such as weights.

(7) Using genetic algorithm to the optimal path selection algorithm for path optimization.

(8) The optimization results back to the output interface, determine whether the current vehicle position have to the finish line at the same time, is to stop counting; Otherwise the step (9).

(9) Accept new real-time traffic information from the transportation center, the current vehicle location, at the current vehicle position as a starting point, the finish is changeless, step (2).

\section{Simulation experiment and result analysis}

Shenyang municipal level within a ring trunk road network is as the research object in the simulation. The road network includes 74 nodes, 262 have to stretch. Among them, the linear distance between two points of the road network furthest distance of $10.98 \mathrm{~km}$.

Improve the optimal dynamic vehicle routing algorithm simulation experiment was carried out using MATLAB simulation language, each time the beginning and end of the simulation of random generation, remove the part of the same simulation results, among them 500 times average optimization simulation run time for $0.1-1 \mathrm{~s}$, efficiency reached $99.8 \%$, with $97.6 \%$ accuracy. Therefore, the improved optimal path selection algorithm can meet the real-time demand in route guidance of intelligent traffic.

\section{IV.CONCLUSIONS}

This paper adopted the optimal path selection algorithm based on genetic algorithm; it is verified by simulation experiments for the effectiveness of the optimal route guidance traffic system calculation. At the same time is proposed in this paper based on the rectangle restricted areas search algorithm and genetic algorithm combining dynamic route guidance algorithm. Finally, the simulation experiments prove the validity of the method used in largescale traffic network with real-time, and have certain application value.

\section{REFERENCES}

[1] LIAKOU, HARIS Marina, "An overview on transports and ITS," Communication in Computer and information science. Berlin, vol.112, 2010, pp. 343-345.

[2] National Bureau of Statistics of China,"China Statistical Yearbook 2014,"China Statistical Press. Beijing, 2012, pp. 16-44.

[3] LIU Xiao ming, "Urban Transportation and Management: The Scientific Development of Urban Transportation in China," Journal of Transportation Systems Engineering and Information Technology. Beijing, vol.10,2010, pp. 11-21.

[4] Downs A, "Can traffic congestion be cured?" The Washington Post. Washington, vol.4,2006,pp. 955-974.

[5] Wang Xin, Huang Kuan, Gao Zhijun. The Primary Users' Signals Recognition Algorithm in Cognitive Radio Networks via KPCA and Random Forest[J].ICIC Express Letters, 2015, 9(4):1083-1088.

[6] MA Yongjie,YUN Wenxia, "Research progress of genetic algorithm," Application Research of Computers. Chengdu,vol.29, 2012,pp. 1201-1206.

[7] Ge JiKe, Qiu YuHui, Wu ChunMing, "Summary of genetic algorithms research," Application Research of Computers. Chengdu, vol.25, 2008,pp. 2911-2916.

[8] HE Yao-hua, HUI Chi-wai, "A binary coding genetic algorithm for multi-purpose process scheduling: a case study," Chemical Engineering Science. America, vol.65, 2010,pp.4816- 4828.

[9] BORIS P L, JESSICA S C, “A deterministic annular crossover genetic algorithm optimization for the unit commitment problem ," Expert Systems with Applications. Kidlington, vol.38, 2011, pp.6523-6529.

[10] Wang Xin,Guo Lili,Ma Lina. Cooperative Spectrum Sensing Algorithm Based on Second User Selection and Random Forest Classification[J].ICIC Express Letters, Part B: Applications,2015, 6(3):845-850.

[11] Lu Feng, Cui Weihong, Lu Dongmei, "Time Shortest Path Algorithm for Restricted Searching Area in Transportation Networks ," Journal of Image and Graphics. Beijing, vol.4,1999, pp. 849-853. 